\title{
La vía de la insulina y el factor de crecimiento similar a la insulina, una nueva diana terapéutica en oncología
}

\author{
Insulin and insulin-like growth factor pathway, a new targeted \\ therapy in oncology
}

\author{
J. Bosch-Barrera, A. Hernández, L.E. Abella
}

\section{RESUMEN}

La biología molecular del cáncer ha permitido identificar nuevas dianas para atacar las células tumorales. Recientemente se ha propuesto la vía de señalización de la insulina y el factor de crecimiento similar a la insulina como una de estas dianas. En esta revisión se describe su función biológica, los datos de laboratorio y estudios poblacionales que alertan de su papel en el cáncer y se describen los elementos claves de esta vía de señalización: los ligandos (insulina, IGF1, IGF2), sus receptores y la cascada de señales intracelular que desencadena su activación. Así mismo se revisan las distintas estrategias que se están investigando para bloquearla, algunas de las cuales ya se encuentran en estudios avanzados fase III. Los datos preliminares indican que los fármacos diseñados para bloquear esta vía pueden ser una nueva arma terapéutica para los pacientes oncológicos en un futuro próximo.

Palabras clave. Factor de crecimiento similar a la insulina I. Factor de crecimiento similar a la insulina II. Receptor de la insulina. Receptores relacionados con el receptor de la insulina.

\begin{abstract}
The molecular biology of cancer has made it possible to identify new targets for attacking tumourous cells. One of these recently proposed targets is the insulin and insulin-like growth factor signaling pathway. This review describes its biological function, laboratory data, population studies that warn of its role in cancer, and the key elements of this signaling pathway: the ligands (insulin, IGF1, IGF2), its receptors and the cascade of intracellular signals that trigger its activation. Also reviewed are the different strategies under investigation for blocking it, some of which are already in phase III advanced studies. The preliminary data indicate that the medicines designed for blocking this pathway might be a new therapeutic weapon for oncology patients in the near future.
\end{abstract}

Key words. Insulin-like growth factor II. Insulin-like growth factor I. Insulin receptor. Insulin receptor-related receptor.

An. Sist. Sanit. Navar. 2009; 32 (3): $413-421$

Departamento de Oncología médica. Clínica Universidad de Navarra. Pamplona

Recepción: 10 de abril de 2009

Aceptación provisional: 15 de junio de 2009

Aceptación definitiva: 1 de julio de 2009

\author{
Correspondencia \\ Joaquim Bosch-Barrera \\ Departamento de Oncología \\ Clínica Universidad de Navarra \\ Avda. Pío XII, 36 \\ 31008 Pamplona \\ Tfno. 948255400 \\ E-mail: jbosch@unav.es
}




\section{INTRODUCCIÓN}

En los últimos años se ha realizado un importante avance en el tratamiento del cáncer mediante la optimización de los tratamientos quirúrgicos, radioterápicos y de fármacos citostáticos. Así mismo, se han incorporado al arsenal terapéutico disponible para los oncólogos las llamadas terapias biológicas o dirigidas contra el cáncer. Se caracterizan por ser productos que actúan específicamente sobre alguna diana molecular importante en los mecanismos implicados en la proliferación, supervivencia, angiogénesis, capacidad de invasión o capacidad de generar metástasis de las células malignas.

Algunas de estas terapias dirigidas ya son ampliamente utilizadas en los hospitales tras haber superado las distintas etapas necesarias de investigación básica, diseño farmacológico y ensayos clínicos. Algunos ejemplos de dianas moleculares de las que disponemos actualmente fármacos comercializados son:

- Vía del EGFR (receptor del factor de crecimiento epidérmico): cetuximab, panitumumab, erlotinib y gefinitib.

- Vía del HER-2 (receptor 2 del factor de crecimiento epidérmico humano): trastuzumab, lapatinib.

- Vía de la mTOR (diana de la rapamicina en el mamífero): temsirolimus y próximamente everolimus (aprobado en USA por la Food and Drug Administration).

- Vía del VEGF (factor de crecimiento del endotelio vascular): bevacizumab.

- Pequeñas moléculas inhibidoras de dominios tirosinquinasa: sunitinib, sorafenib.

- Vía del oncogen c-Kit: imatinib mesilato.

Aunque estos avances han supuesto en algunos casos una revolución, sigue siendo todavía una urgencia mejorar el tratamiento del cáncer. La investigación de la biología molecular de la célula tumoral nos ha permitido identificar una nueva posible diana susceptible de generar nuevos fármacos: la vía de señalización de la insulina y el factor de crecimiento similar a la insulina.

En este trabajo nos proponemos revisar la importancia de esta vía, sus elementos claves y algunas de las evidencias sobre su implicación en el cáncer. Finalmente revisaremos algunas de las moléculas que se están ensayando para bloquearla y que, si los estudios clínicos en marcha son positivos, permitirán poder disponer de ellas en la labor asistencial próximamente.

\section{LA VÍA DE LA INSULINA Y LA PROLIFERACIÓN CELULAR}

El papel de la vía relacionada con la insulina es sin duda muy importante en la fisiología celular, como muestra el hecho de su presencia en organismos primitivos. Son varios los organismos invertebrados que ya presentan un receptor similar a la insulina como la $D$. melanogaster y el C. elegans ${ }^{1}$.

Así, por ejemplo, las investigaciones han mostrado que en el $C$. elegans las moléculas relacionadas con el receptor de la insulina y el receptor del factor de crecimiento similar a la insulina-1 o IGFR1 (insulin-like growth factor 1 receptor) desarrollan un papel importante en la regulación celular en función de la disponibilidad de nutrientes en su entorno ${ }^{2}$. Así mismo, el receptor de la insulina o IR (insulin receptor) es necesario para la embriogénesis adecuada en el pez cebra o Brachidanio rerio ${ }^{3}$. Por todos estos motivos, la vía de señalización de la insulina y del factor de crecimiento de la insulina o IGF ha despertado el interés científico, ya que podría ser una diana molecular interesante para luchar contra el cáncer. Actualmente existen ensayos clínicos con más de 10 fármacos distintos cuyo objetivo común es bloquear la vía de señalización del IGF1, algunos de los cuales se encuentran ya en fase III.

Otras evidencias indirectas de su papel en el cáncer son los resultados de estudios poblacionales que han relacionado el uso de algunos fármacos antidiabéticos orales, como es la metformina, con una 
disminución de la incidencia y mortalidad del cáncer ${ }^{4}$.

La vía de señalización de la insulina y del IGF presenta una peculiaridad importante en cuanto a otras vías que se han estudiado hasta la fecha, como puede ser la vía del EGFR ${ }^{5}$, y es que esta vía tiene un papel endocrino además del papel que puede desarrollar en la fisiología celular. Así pues, la insulina y el IGF actúan como factores de crecimiento celulares, pero también desarrollan una función hormonal regulando el crecimiento y metabolismo a nivel sistémico. No hay que olvidar que estas moléculas fueron inicialmente estudiadas y descritas por su función endocrina antes de conocerse su papel regulador celular ${ }^{6}$. Nos centraremos en nuestra revisión en el papel que desarrollan la insulina y el IGF y sus receptores en la fisiología celular y, en especial, en el cáncer.

\section{CONOCIENDO A LOS PROTAGONISTAS}

A continuación presentaremos uno a uno los distintos "actores" de la vía de señalización de la insulina y del IGF para poder entender mejor como interactúan y regulan los procesos celulares (Fig. 1).

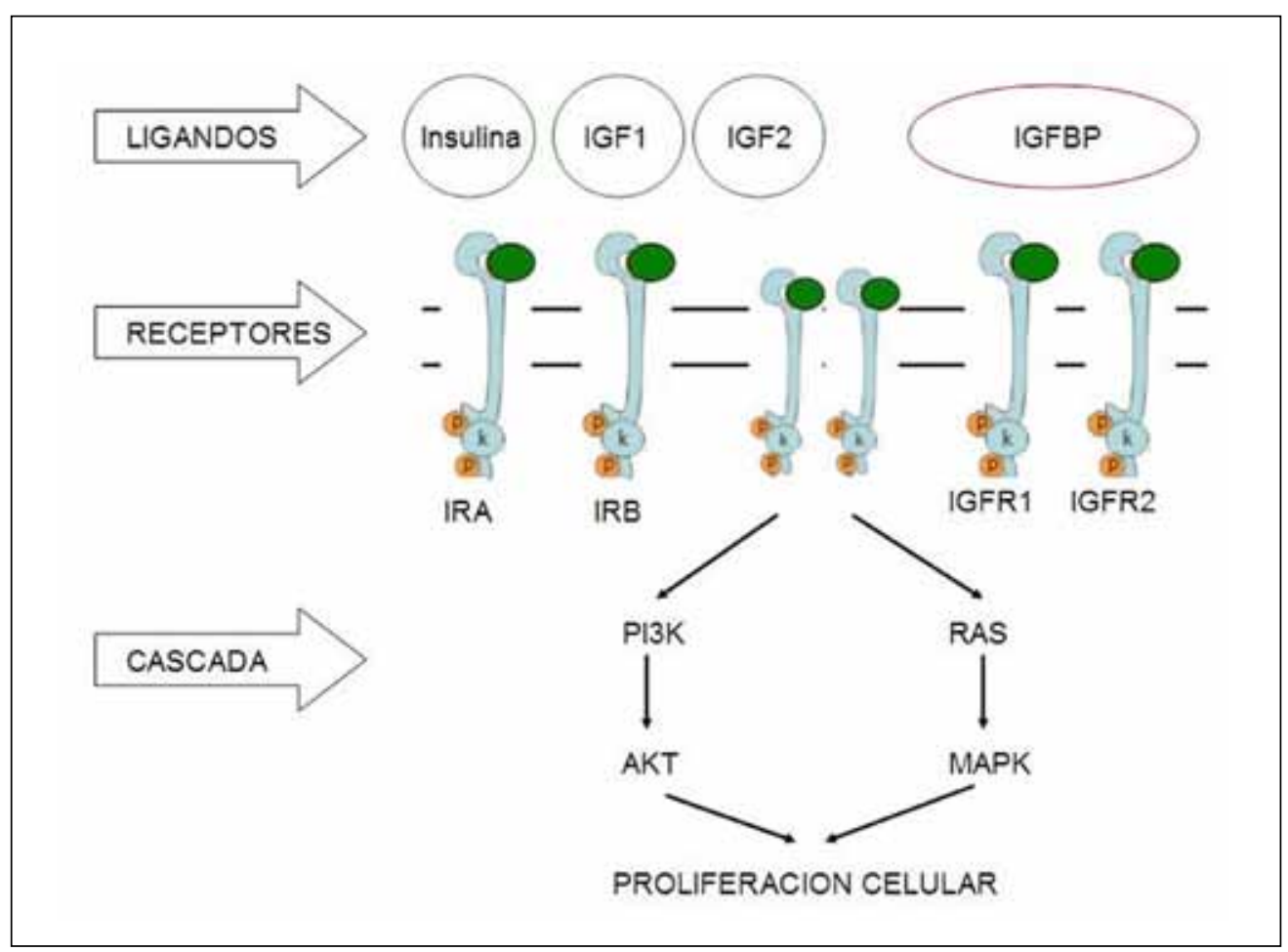

Figura 1. Ligandos de la vía (insulina, IGF1, IGF2) y proteínas de unión al IGF (IGFBP) que modulan su actividad. A nivel de la membrana celular se encuentran los distintos receptores (IRA, IRB, IGFR1, IGFR2), los cuales deben dimerizarse, pudiéndolo hacer entre ellos en distintas combinaciones incluyendo homo o heterodímeros (IRA-IRA / IRB-IRB / IRA-IRB / IGFR1-IGFR1 / IGFR1-IRA / IGFR1-IRB). El IGFR2 no realiza transducción de señales. El resto de combinaciones, una vez activados, mediante la vía del PI3K-AKT y del RAS-MAPK actúan regulando los procesos moleculares que controlan en último término la proliferación celular. 


\section{Los ligandos}

Son las substancias que se unen a los receptores:

- La insulina: suele ser producida normalmente por la célula beta del páncreas y es distribuida de forma endocrina por la sangre al resto del organismo. Su función sería principalmente la regulación del metabolismo de los carbohidratos.

- El IGF-1 e IGF-2: fueron descritos en 1957 por Salmon y Daughaday ${ }^{7}$. Son principalmente generados en el híga$\mathrm{do}^{8}$, aunque también pueden sintetizarse en el músculo esquelético ${ }^{9}$, y a través del torrente circulatorio pueden actuar de un modo sistémico. Sin embargo, también existe una producción de estos ligandos en los propios tejidos tumorales. Por este motivo, se ha descrito actuación autocrina, paracrina y endocrina con estas moléculas. Su función sería principalmente el control de la proliferación celular.

\section{Las proteínas de unión}

El IGF-1 y el IGF-2 pueden ser también regulados por las proteínas de unión o BPs (binding proteins), que tienen afinidad para ambos ligandos. Generalmente, estas proteínas de unión del IGF (IGFBPs) actúan atenuando la actividad del IGF, ya que evitan que pueda unirse al IGFR $1^{10}$. No es de extrañar, por tanto, que algunos genes supresores tumorales como p53 sean capaces de aumentar la producción y secreción de IGFBPs ${ }^{11}$. Sin embargo, esto no es tan sencillo, ya que algunas IGFBPs (particularmente IGFBP2 y IGFBP5) se han asociado a un aumento de la actividad de IGF, con peor supervivencia y pérdida de función de $\mathrm{PTEN}^{12}$. Finalmente, no se descarta que las IGFBPs puedan regular la actividad celular independiente de su capacidad de unirse al IGF, punto que está siendo motivo de investigaciones en el momento actual ${ }^{13}$.

\section{Los receptores}

Existen principalmente dos tipos de receptores. Por un lado, están los receptores de la insulina que incluyen la isoforma IRA (más expresada por las células tumorales) y la isoforma IRB (habitualmente expresada por los tejidos clásicamente sensibles a la insulina en los adultos) que son generados por splicing alternativo. Por otro lado, están los receptores del IGF (IGFRs) ${ }^{14,15}$.

Todos estos receptores son estructuras tetraméricas, caracterizados por dos "medio-receptores" cada uno de los cuales a su vez comprende una cadena predominantemente alfa extracelular que conforma el punto de unión al ligando y una cadena predominantemente beta intracelular que posee el dominio tirosinquinasa necesario para la transducción de señales ${ }^{16}$. En las células pueden aparecer receptores homo o heteropolimerizados entre las distintas subunidades del receptor de insulina e IGFRs, causando la aparición de receptores híbridos ${ }^{14}$. La distinta afinidad de los receptores a sus ligandos, así como la especificidad de la actividad tirosinquinasa de los mismos, puede contribuir a modular aún más la actividad de esta vía en cada célula o tejido.

Finalmente, existe un receptor específico para el IGF2 cuya función es actuar como un quelante del IGF2, ya que al unirse el ligando no transmite señales intracelulares y actuaría por tanto como un gen supresor de tumores ${ }^{17}$.

\section{La cascada intracelular}

La cascada intracelular es una vía compleja ya que hay diversos ligandos activos y diversos receptores, que incluso se pueden combinar entre ellos ampliando aún más la complejidad de su acción.

Una vez unido el ligando adecuado en el complejo receptor (ya sea por los receptores de la insulina, los IGFRs o los híbridos de ambos), el receptor activaría la cascada de señales intracelulares, mediante las vías bien conocidas del fosfatidilinositol-trifosfato quinasa (PI3K) que activa a su vez al AKT o bien por la vía de la Ras-MAP quinasa. Mediante estas cascadas de señales se produciría un aumento de la proliferación celular y un estímulo antiapoptótico ${ }^{18}$. 


\section{La vía de la insulina y el IGF en el cáncer}

Algunos estudios iniciales sobre el papel de la insulina como factor de crecimiento celular mostraron que está relacionada con la estimulación de la síntesis de ADN en las células de cáncer de mama ${ }^{19}$ y que la deficiencia de insulina se asocia con una proliferación in vivo del cáncer menos agresiva $^{20}$. Sin embargo, las dudas acerca del efecto sobre el organismo que podría suponer bloquear la señalización de la insulina a nivel sistémico hicieron caer en el olvido estos datos empíricos.

La identificación en 1987 del IGFR1 abrió nuevas perspectivas ${ }^{21}$, ya que se fue descubriendo el papel central que desarrolla en el cáncer al verse que dosis crecientes de IGF1 aumentaban la proliferación tumoral o que la señalización a través de IGF1 facilitaba la activación de oncogenes ${ }^{22-23}$. Así, en el estudio de Myal y col, cuando se estimuló un modelo experimental de células de cáncer de mama humano (T-47D) con 1 microgramo/litro de IGF-I e IGF-II en un cultivo sobre plástico, se produjo un aumento de 1,5 veces la proliferación celular. Cuando la dosis de estos factores se subió a 500 $\mathrm{ng} / \mathrm{ml}$ la magnitud del efecto fue de 4 veces para el IGF-II, de 2,5 veces para el IGF-I y de 1,5 veces para la insulina.

También distintos estudios epidemiológicos mostraron que los individuos con diagnóstico previo de cáncer de próstata, mama, colorrectal u otros tumores que presentaban límites altos de los niveles séricos de IGF1 tenían más del doble de riesgo de desarrollar otro cáncer que los que presentaban niveles bajos o normales de este marcador sérico ${ }^{24,25}$. A partir de estos datos se fue concibiendo el papel del IGFR1 como posible diana terapéutica ${ }^{26}$.

\section{TERAPIAS DIRIGIDAS CONTRA LA VÍA DE LA INSULINA Y EL IGF}

No es de extrañar, como hemos dicho, que tras estos datos empíricos que mostraban el papel de la vía de la insulina y el IGF en el cáncer se diseñaran estrategias para bloquearla como forma de atacar las células tumorales. Existen distintos puntos de la vía en los que actuar y han ido apareciendo gracias a la investigación molecular y farmacéutica distintos productos, de los que revisaremos los principales.

\section{Bloquear al ligando}

El bloqueo del ligando fue la primera estrategia que se propuso mediante la utilización de los análogos de la somatostatina para reducir los niveles circulantes de IGF1, aunque fueron infructuosos ${ }^{27}$. A pesar de estos resultados negativos iniciales, se han realizado otras propuestas en este sentido como anticuerpos específicos contra el ligando o antagonistas de la hormona del crecimiento con resultados preclínicos interesantes. Así, Goya y col utilizaron el KM1468 (anticuerpo contra IGF-I e IGF-II) en un modelo murino mostrando su utilidad para evitar la formación y progresión de metástasis óseas ${ }^{28}$. Otra experiencia en este sentido es la de Divisova y col, esta vez utilizando el pegvisomant (un antagonista de la $\mathrm{GH}$ ), mostrando que su administración intraperitoneal suprimía en un $60 \%$ los niveles hepáticos de RNA mensajero hepático y un 70-80\% los niveles séricos de IGF-I. Este efecto del pegvisomant se tradujo en una respuesta en los xenoinjertos MCF-7 de cáncer de mama en ratones, aunque no fue eficaz en los xenoinjertos MDA231 o MDA- $435^{29}$.

\section{Bloquear al receptor extracelularmente}

El dominio extracelular del receptor es una diana interesante para generar anticuerpos monoclonales, como ha ocurrido en otro tipo de dianas moleculares como el HER-2 (trastuzumab) o el EGFR (cetuximab, panitumumab).

Existen distintos anticuerpos contra el IGFR1 que se han estudiado en ensayos preclínicos. Actualmente el que se conoce con más detalle es el CP-751871 30,31 . La toxicidad con este anticuerpo ha sido aceptable, siendo su principal beneficio el aumento de 
la tasa de respuestas en combinación con quimioterapia en pacientes con carcinoma no microcítico de pulmón, especialmente en los subtipos histológicos de carcinoma escamoso. En el Congreso de la Asociación de la Sociedad Americana de Oncología (ASCO) del año 2008 se comunicaron los resultados preliminares del estudio fase III que analiza el esquema de quimioterapia estándar de taxol $(200 \mathrm{mg} / \mathrm{m} 2)$ y carboplatino (AUC de 6) con o sin CP-751871 en carcinoma no microcítico de pulmón. En ese momento se disponía de la información de 143 pacientes para el objetivo primario que era tasa de respuestas objetivas según criterios RECIST. Se apreció con este análisis una mejoría significativa a favor del grupo experimental ( $51 \%$ vs $36 \%, \mathrm{p}<0,001)$. Cuando se analizó el subgrupo de paciente con histología escamosa esta diferencia fue aún más llamativa ( $72 \%$ vs $42 \%, \mathrm{p}<0,001)$. En cuanto a la toxicidad, se pudieron analizar en ese momento los datos de 178 pacientes, comunicándose una toxicidad grado 3-4 de hiperglucemia ( $11 \%$ vs $4 \%$ ), astenia ( $9 \%$ vs $7 \%$ ) y neutropenia ( $14 \%$ vs $14 \%$ ) como toxicidades más reseñables ${ }^{32}$. Otros anticuerpos de distintas compañías farmacéuticas que están siendo estudiados actúan contra el IGFR1 con un perfil de tolerancia similar al CP-751871 ${ }^{33-37}$. Se ha detectado en estos estudios clínicos la presencia de un aumento de IGF1 y hormona del crecimiento circulante que era previsible, así como una hiperglicemia ${ }^{31,38,39}$. No hay evidencia a día de hoy de que el aumento de IGF1 pueda superar el bloqueo que supone el anticuerpo en el IGFR1 que pudiera contrarrestar el beneficio de este tratamiento de anticuerpo monoclonal.

\section{Bloquear al receptor intracelularmente}

El dominio intracelular tirosinquinasa también ha sido la diana molecular de algunos fármacos en otras vías de señalización celular como por ejemplo en la vía del HER2 (lapatinib) o del EGFR (gefitinib, erlotinib).

En el caso de la vía de la insulina y del IGF estos fármacos se encuentran en una fase muy precoz de su desarrollo, habiéndose realizado únicamente ensayos clínicos fase I de algunos de ellos ${ }^{40-45}$.

\section{Metformina}

Algunos estudios epidemiológicos mostraron el papel protector de la metformina en la prevención del cáncer ${ }^{4}$. Se considera a este fármaco como un sensibilizador de la insulina (actuando como un antidiabético oral), aunque actualmente se sugiere que es un activador de la vía AMPK-LKB1.

En el hígado la activación de la vía AMPK-LKB1 inhibe la gluconeogénesis y la salida de glucosa del hígado, disminuyendo por tanto el nivel de glucemia y en consecuencia el nivel de insulinemia ${ }^{46}$. A nivel celular, se ha visto que en las células epiteliales transformadas actuaría como un inhibidor de la proliferación ${ }^{47,48}$. Así pues, la metformina puede estar actuando desde un punto de vista endocrino disminuyendo la cantidad de ligando disponible en plasma, y desde un punto de vista molecular mediante la vía AMPK-LKB1 inhibiendo la cascada de la mTOR relacionada con la síntesis proteica, biogénesis de ribosomas, transcripción y organización actínica.

\section{CONSIDERACIONES FINALES}

La vía de señalización de la insulina y el IGF tiene un importante papel en las células cancerígenas a raíz de los datos de laboratorio y estudios poblacionales realizados hasta la fecha. Se conocen varios ligandos de la vía (insulina, IGF1, IGF2), que actúan en distintos receptores de la insulina (isoformas IRA, IRB) y distintos IGFRs (IGFR1, IGFR2) que además pueden ser híbridos entre ellos. Una vez activados los receptores, la transducción de señales se realiza a través de la vía PI3K-Akt y RAS-MAPK promoviendo la proliferación celular y mecanismos antiapoptóticos. Sin embargo, no hay que olvidar que el IGFR2 no presenta actividad intracelular comportándose como un gen supresor de tumores.

Esta vía se ha convertido en una diana terapéutica interesante, habiéndose dise- 
ñado varias estrategias para bloquearla, ya sea actuando en el ligando, en el dominio extracelular o intracelular del receptor o con fármacos antidiabéticos capaces de disminuir los niveles circulantes de ligandos y activando la vía AMPK-LKB que bloquea a la vía de la mTOR. Los datos preliminares obtenidos con varios de estos fármacos indican que el bloqueo de esta vía puede ser una nueva arma terapéutica disponible para los pacientes oncológicos en un futuro próximo.

\section{BIBLIOGRAFÍA}

1. Chan SJ, Steiner DF. Insulin through the ages: phylogeny of a growth promoting and metabolic regulatory hormone. American Zoologist 2000; 40: 213-222.

2. Dong MQ, Venable JD, Au N, Xu T, Park SK, CoCIORVA D et al. Quantitative mass spectrometry identifies insulin signaling targets in C. elegans. Science 2007; 317: 660-663.

3. Toyoshima Y, Monson C, Duan C, Wu Y, Gao C, YAKAR $S$ et al. The role of insulin receptor signaling in zebrafish embryogenesis. Endocrinology 2008; 149: 5996-6005.

4. Evans JM, Donnelly LA, Emsle-Smith AM, Alessi DR, MorrIS AD. Metformin and reduced risk of cancer in diabetic patients. BMJ 2005; 330 : 1304-1305.

5. BuRGESS AW. EGFR family: structure physiology signalling and therapeutic targets. Growth Factors 2008; 26: 263-274.

6. RAJU TN. A mysterious something: the discovery of insulin and the 1923 Nobel Prize for Frederick G. Banting (1891-1941) and John J.R. Macleod (1876-1935). Acta Paediatr 2006; 95: 1155-1156.

7. SAlmon WD JR, Daughaday W. A hormonally controlled serum factor which stimulates sulfate incorporation by cartilage in vitro. $\mathrm{J}$ Lab Clin Med 1957; 49: 825-836

8. LARON Z. The somatostatin-GHRH-GH-IGF-I axis. En: Merimee T, Laron Z, eds. Growth hormone, IGF-I and growth: new views of old concepts. Modern endocrinology and diabetes, Vol. 4. London-Tel Aviv: Freund Publishing House Ltd, 1996: 5-10.

9. Berg U, BAng P. Exercise and circulating insulin-like growth factor I. Horm Res 2004; 62 Suppl. 1: 50-58.

10. Firth SM, BaXter RC. Cellular actions of the insulin-like growth factor binding proteins. Endocr Rev 2002; 23: 824-854.
11. Buckbinder L, Talbott R, Velasco-Miguel S, TakeNAKA I, FAHA B, SEIZINGER BR et al. Induction of the growth inhibitor IGF-binding protein 3 by p53. Nature 1995; 377: 646-649.

12. Mehrian-Shai R, Chen CD, Shi T, Horvath S, NelSON SF, REICHARDT JK et al. Insulin growth factor-binding protein 2 is a candidate biomarker for PTEN status and PI3K/Akt pathway activation in glioblastoma and prostate cancer. Proc Natl Acad Sci U S A 2007; 104: 55635568.

13. Zhu W, Shiojima I, Ito Y, Li Z, Ikeda H, Yoshida M et al. IGFBP-4 is an inhibitor of canonical Wnt signalling required for cardiogenesis. Nature 2008; 454: 345-349.

14. Belfiore A. The role of insulin receptor isoforms and hybrid insulin/IGF-I receptors in human cancer. Curr Pharm Des 2007; 13: 671686.

15. Frasca F, Pandini G, Sciacca L, Pezzino V, SquaTrito S, Belfiore A et al. The role of insulin receptors and IGF-I receptors in cancer and other diseases. Arch Physiol Biochem 2008; 114: 23-37.

16. De Meyts P. Insulin and its receptor: structure, function and evolution. Bioessays 2004; 26: 1351-1362.

17. De Souza AT, Hankins GR, Washington MK, ORTON TC, JIRTLE RL. M6P/IGF2R gene is mutated in human hepatocellular carcinomas with loss of heterozygosity. Nat Genet 1995; 11 : 447-449.

18. PollaK M. Insulin and insulin-like growth factor signalling in neoplasia. Nat Rev Cancer 2008; 8: 915-928.

19. Osborne CK, Bolan G, Monaco ME, Lippman ME. Hormone responsive human breast cancer in long-term tissue culture: effect of insulin. Proc Natl Acad Sci U S A 1976; 73: 4536-4540.

20. Heuson JC, Legros N, Heimann R. Influence of insulin administration on growth of the 7,12-dimethylbenz(a)anthracene-induced mammary carcinoma in intact, oophorectomized, and hypophysectomized rats. Cancer Res 1972; 32: 233-238.

21. Pollak MN, Perdue JF, Margolese RG, Baer K, RiCHARD M. Presence of somatomedin receptors on primary human breast and colon carcinomas. Cancer Lett 1987; 38: 223-230.

22. Myal Y, Shiu RP, Bhaumick B, Bala M. Receptor binding and growth-promoting activity of insulin-like growth factors in human breast cancer cells (T-47D) in culture. Cancer Res 1984; 44 (12 Pt 1): 5486-5490. 
23. Sell C, Rubini M, Rubin R, Liu JP, Efstratiadis A, BASERGA R. Simian virus 40 large tumor antigen is unable to transform mouse embryonic fibroblasts lacking type 1 insulin-like growth factor receptor. Proc Natl Acad Sci U S A 1993; 90: 11217-11221.

24. Ma J, Pollak MN, Giovannucci E, Chan JM, Tao $\mathrm{Y}$, HeNNEKENS $\mathrm{CH}$ et al. Prospective study of colorectal cancer risk in men and plasma levels of insulin-like growth factor (IGF)-I and IGFbinding protein-3. J Natl Cancer Inst 1999; 91 : 620-625.

25. Giovannucci E, Pollak MN, Platz EA, Willett WC, Stampfer MJ, Majeed N et al. A prospective study of plasma insulin-like growth factor- 1 and binding protein-3 and risk of colorectal neoplasia in women. Cancer Epidemiol Biomarkers Prev 2000: 345-349.

26. Wu Y, Cui K, Miyoshi K, Hennighausen L, Green JE, SETSER J et al. Reduced circulating insulin-like growth factor I levels delay the onset of chemically and genetically induced mammary tumors. Cancer Res 2003; 63: 4384-4388.

27. Ingle JN, Suman VJ, Kardinal CG, Krook JE, Mailliard JA, VeEder MH et al. A randomized trial of tamoxifen alone or combined with octreotide in the treatment of women with metastatic breast carcinoma. Cancer 1999; 85: 1284-1292.

28. Goya M, Miyamoto S, Nagai K, Ohki Y, Nakamura $\mathrm{K}$, Shitara K et al. Growth inhibition of human prostate cancer cells in human adult bone implanted into nonobese diabetic/severe combined immunodeficient mice by a ligand-specific antibody to human insulin-like growth factors. Cancer Res 2004; 64: 62526258.

29. Divisova J, Kuiatse I, Lazard Z, Weiss H, VReEland F, Hadsell DL, et al. The growth hormone receptor antagonist pegvisomant blocks both mammary gland development and MCF-7 breast cancer xenograft growth. Breast Cancer Res Treat 2006; 98: 315-327.

30. Lacy MQ, Alsina M; Fonseca R, Paccagnella ML, Melvin CL, Yin D et al. Phase I, Pharmacokinetic and pharmacodynamic study of the antiinsulin like growth factor type 1 receptor monoclonal antibody CP-751,871 in patients with multiple myeloma. J Clin Oncol 2008; 26 : 3196-3203.

31. Haluska P, Shaw HM, Batzel GN, Yin D, Molina JR, Molife LR et al. Phase I dose escalation study of the anti insulin-like growth factor-I receptor monoclonal antibody CP-751,871 in patients with refractory solid tumors. Clin Cancer Res 2007; 13: 5834-5840.
32. Karp DD, Paz-Ares LG, Novellos S, Haluska P, Garland L, Cardenal F et al. High activity of the anti-IGF-IR antibody CP-751,871 in combination with paclitaxel and carboplatin in squamous NSCLC. J Clin Oncol 2008; 26 (Suppl), 8015.

33. Tolcher AW, Rothenberg ML, Rodon J, Delbeke D, Patnaik A, Nguyen L et al. A phase I pharmacokinetic and pharmacodynamic study of AMG 479, a fully human monoclonal antibody against insulin-like growth factor type 1 receptor (IGF-1R), in advanced solid tumors. J Clin Oncol 200725 (Suppl) 3002.

34. Moreau P, Hulin C, Facon T, Boccadoro M, MeryMignard D, Deslandes A et al. Phase I study of AVE1642 anti IGF-1R monoclonal antibody in patients with advanced multiple myeloma. Proceedings of ASH 2007, abstract 1166.

35. Tolcher AW, Patnaik A, Till E, Takimoto $\mathrm{CH}$, Papadopoulos KP, Massard C et al. A phase I study of AVE1642, a humanized monoclonal antibody IGF-1R antagonist, in patients with advanced solid tumor. J Clin Oncol 2008; 26 (Suppl), 3582.

36. Hidalgo M, Tirado Gómez M, Lewis N, Vuky JL, TAYLOR G, HaYburn JL et al. A phase I study of MK-0646, a humanized monoclonal antibody against the insulin-like growth factor receptor type 1 in advanced solid tumor patients in a q2 wk schedule. J Clin Oncol 2008; 26 (Suppl), 3520.

37. Rodon J, Patnaik A, Stein M, Tolcher A, NG C, DiA C et al. A phase I study of q3W R1507, a human monoclonal antibody IGF-1R antagonist in patients with advanced cancer. J Clin Oncol 2007; 26 (Suppl), 3590.

38. Pollak MN, Schernhammer ES, Hankinson SE. Insulin-like growth factors and neoplasia. Nature Rev Cancer 2004; 4, 505-518.

39. DEL Rincon JP, LidA K, Gaylinn BD, McCuRdy CE, LEITNER JW, BARCOUR LA et al. Growth hormone regulation of p85 expression and phosphoinositide 3-kinase activity in adipose tissue: mechanism for growth hormone-mediated insulin resistance. Diabetes 2007; 56, 1638-1646.

40. Haluska P, Carboni JM, Loegering DA, Lee Fy, WitTMAN M, SAULNIER MG et al. In vitro and in vivo antitumor effects of the dual insulin-like growth factor-I/insulin receptor inhibitor, BMS-554417. Cancer Res 2006; 66: 362-371.

41. Ji QS, Mulvihill MJ, Rosenfeld-Franklin M, CoOKe A, Feng L, MARK G et al. A novel, potent, and selective insulin-like growth factor-I receptor kinase inhibitor blocks insulin-like growth factor-I receptor signaling in vitro and inhibits insulin-like growth factor-I receptor de- 
pendent tumor growth in vivo. Mol Cancer Ther 2007; 6: 2158-2167.

42. Zimmermann K, Wittman MD, Saulnier MG, VelaPARTHI U, LANGLEY DR, SANG X et al. Balancing oral exposure with Cyp3A4 inhibition in benzimidazole-based IGF-IR inhibitors. Bioorg Med Chem Lett 2008; 18: 4075-4080.

43. Mulvihill MJ, Ji QS, CoAte HR, CoOKe A, Dong H, FENG L et al. Novel 2-phenylquinolin-7-yl-derived imidazo[1,5-a]pyrazines as potent insulinlike growth factor-I receptor (IGF-IR) inhibitors. Bioorg Med Chem 2008; 16: 1359-1375.

44. Hofmann F, Garcia-Echeverría C. Blocking the insulin-like growth factor-I receptor as a strategy for targeting cancer. Drug Discov. Today 2005; 10: 1041-1047.

45. Vasilcanu R, Vasilcanu D, Roserngren L, NatalishVILi N, Sehat B, Yin S et al. Picropodophyllin induces downregulation of the insulin-like growth factor 1 receptor: potential mechanistic involvement of Mdm2 and b-arrestin1. Oncogene 2008; 27: 1629-1638.

46. Shaw RJ, Lamia KA, Vasquez D, Koo SH, Bardeesy $\mathrm{N}$, DePINHo RA et al. The kinase LKB1 mediates glucose homeostasis in liver and therapeutic effects of metformin. Science 2005; 310: 1642-1646.

47. Dowling RJ, Zakikhani M, Fantus IG, Pollak M, SONENBERG N. Metformin inhibits mammalian target of rapamycin-dependent translation initiation in breast cancer cells. Cancer Res 2007; 67: 10804-10812.

48. Zakikhani M, Dowling R, Fantus IG, Sonenberg N, PollaK M. Metformin is an AMP kinase-dependent growth inhibitor for breast cancer cells. Cancer Res 2006; 66: 10269-10273. 